CREAT. MATH. INFORM.

Volume 25 (2016), No. 1,

Pages $107-120$
Online version at https : //creative-mathematics. cunbm . utcluj. ro/

Print Edition: ISSN 1584 - 286X; Online Edition: ISSN 1843 - 441X

DOI: https://doi.org/10.37193/CMI.2016.01.14

\title{
Algorithm for Hammerstein equations with monotone mappings in certain Banach spaces
}

\author{
T. M. M. SOW, C. DiOP and N. DJiTTE
}

\begin{abstract}
For $q>1$ and $p>1$, let $E$ be a 2-uniformly convex and $q$-uniformly smooth or $p$ - uniformly convex and 2-uniformly smooth real Banach space and $F: E \rightarrow E^{*}, K: E^{*} \rightarrow E$ be bounded and strongly monotone maps with $D(K)=R(F)=E^{*}$. We construct a coupled iterative process and prove its strong convergence to a solution of the Hammerstein equation $u+K F u=0$. Futhermore, our technique of proof is of independent of interest.
\end{abstract}

\section{INTRODUCTION}

Let $E$ be a real normed space and let $S:=\{x \in E:\|x\|=1\}$. $E$ is said to be smooth if the limit

$$
\lim _{t \rightarrow 0^{+}} \frac{\|x+t y\|-\|x\|}{t}
$$

exists for each $x, y \in S$. $E$ is said to be uniformly smooth if it is smooth and the limit is attained uniformly for each $x, y \in S_{E}$, and $E$ is Fréchet differentiable if it is smooth and the limit is attained uniformly for $y \in S_{E}$.

A normed linear space $E$ is said to be strictly convex if the following holds:

$$
\|x\|=\|y\|=1, x \neq y \Rightarrow\left\|\frac{x+y}{2}\right\|<1 \text {. }
$$

The modulus of convexity of $E$ is the function $\delta_{E}:(0,2] \rightarrow[0,1]$ defined by:

$$
\delta_{E}(\epsilon):=\inf \left\{1-\frac{1}{2}\|x+y\|:\|x\|=\|y\|=1,\|x-y\| \geq \epsilon\right\} .
$$

$E$ is uniformly convex if and only if $\delta_{E}(\epsilon)>0$ for every $\epsilon \in(0,2]$. Let $p>1$. Then $E$ is said to be $p$-uniformly convex if there exists a constant $c>0$ such that $\delta_{E}(\epsilon) \geq c \epsilon^{p}$ for all $\epsilon \in(0,2]$. Observe that every $p$-uniformly convex space is uniformly convex.

Let $E$ be a real normed linear space of dimension $\geq 2$. The modulus of smoothness of $E$, $\rho_{E}$, is defined by:

$$
\rho_{E}(\tau):=\sup \left\{\frac{\|x+y\|+\|x-y\|}{2}-1:\|x\|=1,\|y\|=\tau\right\} ; \quad \tau>0 .
$$

A normed linear space $E$ is called uniformly smooth if

$$
\lim _{\tau \rightarrow 0} \frac{\rho_{E}(\tau)}{\tau}=0 .
$$

It is well known (see, e.g., [1] p.95) that $\rho_{E}$ is nondecreasing. If there exist a constant $c>0$ and a real number $q>1$ such that $\rho_{E}(\tau) \leq c \tau^{q}$, then $E$ is said to be $q$-uniformly smooth.

Received: 19.11.2015. In revised form: 20.12.2015. Accepted: 01.02.2016

2010 Mathematics Subject Classification. 47H04, 47H06, 47H15, 47H17, 47J25.

Key words and phrases. Hammerstein equations, strongly monotone, bounded maps.

Corresponding author: N. Djitte; ngalla.djitte@ugb.edu.sn 
Typical examples of such spaces are the $L_{p}, \ell_{p}$ and $W_{p}^{m}$ spaces for $1<p<\infty$ where, $L_{p}\left(\right.$ or $\left.l_{p}\right)$ or $W_{p}^{m}$ is $\left\{\begin{array}{l}2-\text { uniformly smooth and } p \text { - uniformly convex } \text { if } 2 \leq p<\infty \\ p \text { - uniformly smooth and } 2 \text { - uniformly convex } \text { if } 1<p<2 .\end{array}\right.$

Let $J_{q}$ denote the generalized duality mapping from $E$ to $2^{E^{*}}$ defined by

$$
J_{q}(x):=\left\{f \in E^{*}:\langle x, f\rangle=\|x\|^{q} \text { and }\|f\|=\|x\|^{q-1}\right\}
$$

where $\langle.,$.$\rangle denotes the generalized duality pairing. J_{2}$ is called the normalized duality mapping and is denoted by $J$.

It is well known that $E$ is smooth if and only if $J$ is single valued. Moreover, if $E$ is a reflexive smooth and strictly convex Banach space, then $J^{-1}$ is single valued, one-to-one, surjective and it is the duality mapping from $E^{*}$ into $E$. Finally, if $E$ has uniform Gâteaux differentiable norm, then $J$ is norm-to-weak* uniformly continuous on bounded sets.

Remark 1.1. Note also that a duality mapping exists in each Banach space. We recall from [4] some of the examples of this mapping in $l_{p}, L_{p}, W^{m, p}$-spaces, $1<p<\infty$.

(i) $l_{p}: J x=\|x\|_{l_{p}}^{2-p} y \in l_{q}, \quad x=\left(x_{1}, x_{2}, \cdots, x_{n}, \cdots\right)$,

$$
y=\left(x_{1}\left|x_{1}\right|^{p-2}, x_{2}\left|x_{2}\right|^{p-2}, \cdots, x_{n}\left|x_{n}\right|^{p-2}, \cdots\right) \text {, }
$$

(ii) $L_{p}: J u=\|u\|_{L_{p}}^{2-p}|u|^{p-2} u \in L_{q}$,

(iii) $W^{m, p}: J u=\|u\|_{W^{m, p}}^{2-p} \sum_{|\alpha \leq m|}(-1)^{|\alpha|} D^{\alpha}\left(\left|D^{\alpha} u\right|^{p-2} D^{\alpha} u\right) \in W^{-m, q}$,

where $1<q<\infty$ is such that $1 / p+1 / q=1$.

Let $H$ be a real Hilbert space. A map $G: D(G) \subset H \rightarrow H$ ia called monotone if for all $x, y \in D(G)$, the following inequality holds:

$$
\langle G x-G y, x-y\rangle \geq 0 .
$$

The notion of monotonicity has been extended to real normed spaces $E$ in two ways.

The first involves mapping $G$ from $E$ to $E^{*}$. A map $G: D(G) \subset E \rightarrow E^{*}$ is called strongly monotone if there exists a positive constant $k$ such that for all $x, y \in D(G)$,

$$
\langle G x-G y, x-y\rangle \geq k\|x-y\|^{2},
$$

where $\langle$,$\rangle denotes the duality pairing between elements of E^{*}$ and elements of $E$. The map $G$ is said to be monotone if or all $x, y \in D(G)$,

$$
\langle G x-G y, x-y\rangle \geq 0 .
$$

If $E$ is a real Hilbert space $H$, then $H=H^{*}$ and (1.3) coincides with (1.1).

The second extension of the notion of monotonicity to real normed spaces involves mapping $G$ of $E$ into itself. A mapping $G: D(G) \subset E \rightarrow E$ is called accretive if for every $x, y \in D(G)$, there exists $j(x-y) \in J(x-y)$ such that the following inequality holds:

$$
\langle G x-G y, j(x-y)\rangle \geq 0 .
$$

Here, if $E$ is a real Hilbert space, $J$ becomes the identity map and condition (1.4) reduces to (1.1). Hence, in real Hilbert spaces, accretive operators become monotone. Consequently, accretive operators can be regarded as extension of Hilbert space monotonicity condition to real normed spaces. 
A mapping $G: D(G) \subset E \rightarrow E$ is called strongly accretive if there exists a constant $k>0$ such that for every $x, y \in D(G)$, there exists $j(x-y) \in J(x-y)$ such that

$$
\langle G x-G y, j(x-y)\rangle \geq k\|x-y\|^{2} .
$$

The accretive operators were introduced independently in 1967 by Browder [9] and Kato [31]. Interest in such mappings stems mainly from their firm connection with equations of evolution. It is known (see, e.g., Zeidler [38]) that many physically significant problems can be modelled by initial-value problems of the form:

$$
\frac{d u}{d t}+A u=0, u(0)=u_{0}
$$

where $A$ is an accretive operator in an appropriate Banach space. Typical examples where such evolution equations occur can be found in the heat, wave or Schrödinger equations. If in (1.5), $u(t)$ is independent of $t$, then (1.5) reduces to

$$
A u=0,
$$

whose solutions correspond to the equilibrium points of the system (1.5). Consequently, considerable research efforts have been devoted, especially within the past 40 years or so, to methods of finding approximate solutions (when they exist) of equation (1.6). An early fundamental result in the theory of accretive operators, due to Browder [9], states that the initial value problem (1.5) is solvable if $A$ is locally Lipschitzian and accretive on $E$. Utilizing the existence result for equation (1.5), Browder [9] proved that if $A$ is locally Lipschitzian and accretive on $E$, then $A$ is $m$-accretive i.e, $R(I+A)=E$. Clearly, a consequence of this is that the equation

$$
u+A u=0
$$

has a solution.

One important generalization of equation (1.7) is the so-called equation of Hammerstein type (see, e.g., Hammerstein [29]), where a nonlinear integral equation of Hammerstein type is one of the form:

$$
u(x)+\int_{\Omega} \kappa(x, y) f(y, u(y)) d y=h(x),
$$

where $d y$ is a $\sigma$-finite measure on the measure space $\Omega$; the real kernel $\kappa$ is defined on $\Omega \times \Omega, f$ is a real-valued function defined on $\Omega \times \mathbb{R}$ and is, in general, nonlinear and $h$ is a given function on $\Omega$. If we now define an operator $K: \mathcal{F}(\Omega, \mathbb{R}) \rightarrow \mathcal{F}(\Omega, \mathbb{R})$ by

$$
K v(x):=\int_{\Omega} \kappa(x, y) v(y) d y, \text { a.e. } x \in \Omega
$$

and the so-called superposition or Nemytskii $F: \mathcal{F}(\Omega, \mathbb{R}) \rightarrow \mathcal{F}(\Omega, \mathbb{R})$ by

$$
F u(y):=f(y, u(y)) \text { a.e. } y \in \Omega,
$$

where $\mathcal{F}(\Omega, \mathbb{R})$ denotes a space of functions mapping $\Omega$ into $\mathbb{R}$, then, the integral equation (1.8) can be put in operator theoretic form as follows:

$$
u+K F u=0,
$$

where, without loss of generality, we have taken $h \equiv 0$.

Interest in equation (1.9) stems mainly from the fact that several problems that arise in differential equations, for instance, elliptic boundary value problems whose linear parts possess Green's functions can, as a rule, be transformed into the form (1.9). Among these, 
we mention the problem of the forced oscillations of finite amplitude of a pendulum (see, e.g.,Pascali and Sburlan [34], Chapter IV).

Equations of Hammerstein type play a crucial role in the theory of optimal control systems and in automation and network theory (see, e.g., Dolezale [28]).

Several existence and uniqueness theorems have been proved for equations of Hammerstein type (see, e.g., Brézis and Browder ([8], [6], [7]), Browder [9], Browder and De Figueiredo [10], Bowder and Gupta [11], Chepanovich [12], De Figueiredo [25]).

In general, equations of Hammerstein type (1.9) are nonlinear and there is no known method to find closed form solutions for them. Consequently, methods of approximating solutions of such equations are of interest.

In the special case in which the operator $F$ is angle bounded (defined below) and weakly compact, Brézis and Browder [8, 7] proved the strong convergence of a suitably defined Galerkin approximation to a solution of (1.9). Before we state this theorem, we need the following definitions.

Let $H$ be a real Hilbert space. A nonlinear operator $A: H \rightarrow H$ is said to be anglebounded with angle $\beta>0$ if

$$
\langle A x-A z, z-y\rangle \leq \beta\langle A x-A y, x-y\rangle
$$

for any triple of elements $x, y, z \in H$. For $y=z$, inequality (1.10) implies the monotonicity of $A$.

A monotone linear operator $A: H \rightarrow H$ is said to be angle-bounded with angle $\alpha>0$ if

$$
|\langle A x, y\rangle-\langle A y, x\rangle| \leq 2 \alpha\langle A x, x\rangle^{\frac{1}{2}}\langle A y, y\rangle^{\frac{1}{2}}
$$

for all $x, y \in H$. It is known that the two definitions of angle boundedness are equivalent (see Pascali and Sburlan, [34], Ch. IV, p.189).

We now state the theorem of Brézis and Browder referred to above.

Theorem BB (Brézis and Browder, [7]). Let $H$ be a separable real Hilbert space and $C$ be a closed subspace of $H$. Let $K: H \rightarrow C$ be a bounded continuous monotone operator and $F: C \rightarrow H$ be an angle-bounded and weakly compact mapping. For a given $f \in C$, consider the Hammerstein equation

$$
(I+K F) u=f
$$

and its $n$-th Galerkin approximation given by

$$
\left(I+K_{n} F_{n}\right) u_{n}=P^{*} f,
$$

where $K_{n}=P_{n}^{*} K P_{n}: H \rightarrow C_{n}$ and $F_{n}=P_{n} F P_{n}^{*}: C_{n} \rightarrow H$, where the symbols have their usual meanings (see, [34]). Then, for each $n \in \mathbb{N}$, the Galerkin approximation (1.13) admits a unique solution $u_{n}$ in $C_{n}$ and $\left\{u_{n}\right\}$ converges strongly in $H$ to the unique solution $u \in C$ of the equation (1.12).

It is obvious that if an iterative algorithm can be developed for the approximation of solutions of equations of Hammerstein type (1.12), this will certainly be preferred.

We first note that for the iterative approximation of solutions of equations (1.6) and (1.7) (zeros of accretive type operators), the monotonicity/accretivity of $A$ is crucial. The Mann type iteration scheme (see, e.g., Mann [32]) has successfully been employed (see, e.g., the recent monographs of Berinde [5] and Chidume [13] for results obtained within the past 40 years, or so). One drawback of the Mann iterative scheme, however, is that in general, it only yields weak convergence (see, e.g., Matouskova and Reich [33])

All attempts to use the Mann type iteration scheme directly to approximate solutions of equations of Hammertsein type (1.9) did not yield satisfactory results (see Chidume and 
Osilike [20]). The recurrence formulas used in early attempts involved $K^{-1}$ which is also required to be strongly monotone, and this, apart from limiting the class of mappings to which such iterative schemes are applicable, is also not convenient in applications. Part of the difficulty is the fact that the composition of two monotone operators need not be monotone. It suffices to take

$$
K: \mathbb{R}^{2} \longrightarrow \mathbb{R}^{2}, F: \mathbb{R}^{2} \longrightarrow \mathbb{R}^{2}
$$

where

$$
K=\left(\begin{array}{cc}
1 & 2 \\
-2 & 1
\end{array}\right) \text { and } F=\left(\begin{array}{cc}
0 & 1 \\
-1 & 2
\end{array}\right) .
$$

The first satisfactory results on iterative methods for approximating solutions of Hammerstein equations, as far as we know, were obtained by Chidume and Zegeye [24, 23, 22]. Under the setting of a real Hilbert space $H$, for $F, K: H \rightarrow H$, they defined an auxiliary map on the Cartesian product $E:=H \times H, T: E \rightarrow E$ by

$$
T[u, v]=[F u-v, K v+u] .
$$

We note that

$$
T[u, v]=0 \Leftrightarrow u \text { solves (1.9) and } v=F u \text {. }
$$

With this, they were able to obtain strong convergence of an iterative scheme defined in the Cartesian product space $E$ to a solution of Hammerstein equation (1.9). Extensions to a real Banach space setting were also obtained.

Let $X$ be a real Banach space and $K, F: X \rightarrow X$ be accretive type mappings. Let $E:=X \times X$. The same authors (see, [24,23]) defined $T: E \rightarrow E$ by

$$
T[u, v]=[F u-v, K v+u]
$$

and obtained strong convergence theorems for solutions of Hammerstein equations under various continuity conditions in the Cartesian product space $E$.

The method of proof used by Chidume and Zegeye provided the clue to the establishment of the following coupled explicit algorithm for computing a solution of the equation $u+K F u=0$ in the original space $X$. With initial vectors $u_{0}, v_{0} \in X$, sequences $\left\{u_{n}\right\}$ and $\left\{v_{n}\right\}$ in $X$ are defined iteratively as follows:

$$
\begin{aligned}
& u_{n+1}=u_{n}-\alpha_{n}\left(F u_{n}-v_{n}\right), n \geq 0, \\
& v_{n+1}=v_{n}-\alpha_{n}\left(K v_{n}+u_{n}\right), n \geq 0 .
\end{aligned}
$$

where $\left\{\alpha_{n}\right\}$ is a sequence in $(0,1)$ satisfying appropriate conditions. The recursion formulas $\left(^{*}\right)$ and $\left({ }^{*}\right)$ have been used successfully to approximate solutions of Hammerstein equations involving nonlinear accretive-type operators. Following this, Chidume and Djitte studied this explicit coupled iterative algorithms and proved several strong convergence theorems (see, Chidume and Djitte [17], [18] ). For recent results using these recursion formulas, the reader may consult any of the following references Chidume and Djitte [14], [15], [16], Djitte and Sene [26], [27], Chidume and Ofeodu [19], Chidume and Shehu [21] and also Chapter 13 of [13].

For Hammerstein equations involving monotone mappings from $E$ to $E^{*}$, very little has been achieved. Part of the difficulty is that inequalities involving vectors in $E$ do not generally hold in $E^{*}$. For instance, if $E=L_{p}(p>2)$, then $E^{*}=L_{q}$ with $(1<q<2)$ and generally an inequality that holds in $L_{p}(p>2)$ is reversed in $L_{p}(1<p<2)$, (see, e.g., Chidume [13], Chapter 5.) Interestingly enough, almost all the existence theorems proved for Hammerstein equations involve monotone mappings (see, e.g., Brézis and Browder $[8,6,7]$, 
Browder [9], Browder et al. [10], and Browder and Gupta [11]). Consequently, developing iterative methods for approximating solutions of Hammerstein equations involving monotone mappings is of paramount importance. We note that it has been remarked that in dealing with the Nemistkyi operator, which is intimately connected with the Hammertsein integral equation, its properties are distinguished, in applications, according to two important cases: $L_{p}(\Omega)$ spaces, $1<p<\infty$, and $L_{1}(\Omega)$, (see Pascali and Sburlan [34], Chapter IV, pp. 165, 172). Thus, developing iterative methods for approximating solutions of nonlinear Hammerstein integral equations in these cases is of paramount importance.

It is our purpose in this paper to construct a coupled iterative process and prove its strong convergence to a solution of the Hammerstein equation $u+K F u=0$ with Strongly monotone and bounded mappings in certain Banach spaces including $L_{p}$ spaces, $1<p<\infty$ and all Hilbert spaces. Futhermore, our thechnique of proof is of independent of interest.

\section{PRELIMINARIES}

In the sequel, we shall need the following results and definitions.

Theorem 2.1 (H. K. Xu [37]). Let $p>1$ be a given real number. Then the following are equivalent in a Banach space:

(i) $E$ is p-uniformly convex.

(ii) There is a constant $c_{1}>0$ such that for every $x, y \in E$ and $j_{x} \in J_{p}(x)$, The following inequality holds:

$$
\|x+y\|^{p} \geq\|x\|^{p}+p\left\langle y, j_{x}\right\rangle+c_{1}\|y\|^{p} .
$$

(iii) There is a constant $c_{2}>0$ such that for every $x, y \in E$ and $j_{x} \in J_{p}(x), j_{y} \in J_{p}(y)$, the following inequality holds:

$$
\left\langle x-y, j_{x}-j_{y}\right\rangle \geq c_{2}\|x-y\|^{p} .
$$

Theorem 2.2 (H. K. Xu [37]). Let $q>1$ be a given real number. Then the following are equivalent in a Banach space:

(i) $E$ is q-uniformly smooth.

(ii) There is a constant $c>0$ such that for every $x, y \in E$ and $j_{x} \in J_{q}(x)$, The following inequality holds:

$$
\|x+y\|^{q} \leq\|x\|^{q}+q\left\langle y, j_{x}\right\rangle+c\|y\|^{q} .
$$

(iii) There is a constant $c_{2}>0$ such that for every $x, y \in E$ and $j_{x} \in J_{q}(x), j_{y} \in J_{q}(y)$, the following inequality holds:

$$
\left\langle x-y, j_{x}-j_{y}\right\rangle \leq c_{2}\|x-y\|^{q} .
$$

Lemma 2.1. Let $E$ be a smooth and 2-uniformly convex real Banach space. Then $J^{-1}$ is Lipschtz from $E^{*}$ into $E$, i.e., there exists a constant $L>0$ such that for all $u, v \in E^{*}$,

$$
\left\|J^{-1} u-J^{-1} v\right\| \leq L\|u-v\| .
$$

Proof. This follows from inequality (iii) of Theorem 2.1 with $p=2$.

Lemma 2.2 ([2]). Let $p \geq 2$ and $q>1$, and let $X$ be a $p$-uniformly convex and $q$-uniformly smooth real Banach space. The duality mapping $J: X \rightarrow X^{*}$ is Lipschitz on bounded subsets of $X$; that is, for all $R>0$, there exists a positive constant $m_{2}$ such that

$$
\|J x-J y\| \leq m_{2}\|x-y\|,
$$

for all $x, y \in X$ with $\|x\| \leq R,\|y\| \leq R$. 
Let $E$ be a smooth real Banach space with dual $E^{*}$. The function $\phi: E \times E \rightarrow \mathbb{R}$, defined by

$$
\phi(x, y)=\|x\|^{2}-2\langle x, J y\rangle+\|y\|^{2}, x, y \in E,
$$

where $J$ is the normalized duality mapping from $E$ into $E^{*}$, introduced by Alber has been studied by Alber [2], Alber and Guerre-Delabriere [3], Kamimura and Takahashi[30], Reich[35] and a host of other authors. This functional $\phi$ will play a central role in what follows. If $E=H$, a real Hilbert space, then equation (2.15) reduces to $\phi(x, y)=\|x-y\|^{2}$ for $x, y \in H$. It is obvious from the definition of the function $\phi$ that

$$
(\|x\|-\|y\|)^{2} \leq \phi(x, y) \leq(\|x\|+\|y\|)^{2} \forall x, y \in E .
$$

Let $V: E \times E^{*} \rightarrow \mathbb{R}$ be the functional defined by:

$$
V\left(x, x^{*}\right)=\|x\|^{2}-2\left\langle x, x^{*}\right\rangle+\left\|x^{*}\right\|^{2}, \forall x \in E, x^{*} \in E^{*} .
$$

Then, it is easy to see that

$$
V\left(x, x^{*}\right)=\phi\left(x, J^{-1} x^{*}\right) \forall x \in E, x^{*} \in E^{*} .
$$

Similarly, if $E$ is a reflexive smooth and strictly convex real Banach space, we introduce the functional $\phi_{*}: E^{*} \times E^{*} \rightarrow \mathbb{R}$, defined from by:

$$
\phi_{*}\left(x^{*}, y^{*}\right)=\left\|x^{*}\right\|^{2}-2\left\langle x^{*}, J^{-1} y^{*}\right\rangle+\left\|y^{*}\right\|^{2}, x^{*}, y^{*} \in E^{*},
$$

and the functional $V_{*}: E^{*} \times E \rightarrow \mathbb{R}$ defined from $E^{*} \times E$ to $\mathbb{R}$ by:

$$
V_{*}\left(x^{*}, x\right)=\left\|x^{*}\right\|^{2}-2\left\langle x^{*}, x\right\rangle+\|x\|^{2}, x \in E, x^{*} \in E^{*} .
$$

It is easy to see that

$$
V_{*}\left(x^{*}, x\right)=\phi_{*}\left(x^{*}, J x\right) \forall x \in E, x^{*} \in E^{*} .
$$

In what follows, the product space $E \times E^{*}$ is equiped with the following norm:

$\left\|w_{1}-w_{2}\right\|=\left(\|x-y\|^{2}+\left\|x^{*}-y^{*}\right\|^{2}\right)^{\frac{1}{2}} \forall w_{1}=\left(x, x^{*}\right) \in E \times E^{*}, w_{2}=\left(y, y^{*}\right) \in E \times E^{*}$.

Finally, we introduce on the functional $\psi:\left(E \times E^{*}\right) \times\left(E \times E^{*}\right) \rightarrow \mathbb{R}$ defined by:

$$
\psi\left(w_{1}, w_{2}\right):=\phi(x, y)+\phi_{*}\left(x^{*}, y^{*}\right) \forall w_{1}=\left(x, x^{*}\right) \in E \times E^{*}, w_{2}=\left(y, y^{*}\right) \in E \times E^{*} .
$$

The following results will be useful.

Lemma 2.3 (Alber, [2]). Let E be a reflexive striclty convex and smooth real Banach space with $E^{*}$ as its dual. Then,

$$
V\left(x, x^{*}\right)+2\left\langle J^{-1} x^{*}-x, y^{*}\right\rangle \leq V\left(x, x^{*}+y^{*}\right)
$$

for all $x \in X$ and $x^{*}, y^{*} \in X^{*}$.

Lemma 2.4. Let $E$ be a smooth and 2-uniformly convex real Banach space. Then there exists $c_{1}>0$ such that

$$
\|x-y\|^{2} \geq \phi(x, y)+\left(c_{1}-1\right)\|x\|^{2} \forall x, y \in E .
$$

Proof. From Theorem 2.1, we have

$$
\|x+y\|^{2} \geq\|x\|^{2}+2\langle y, J x\rangle+c_{1}\|y\|^{2} .
$$

Replacing $y$ by $-y$, we obtain

$$
\|x-y\|^{2} \geq\|x\|^{2}-2\langle y, J x\rangle+c_{1}\|y\|^{2} .
$$


Interchanging $y$ and $x$, we obtain

$$
\begin{aligned}
\|x-y\|^{2} & \geq\|y\|^{2}-2\langle x, J y\rangle+c_{1}\|x\|^{2} \\
\|x-y\|^{2} & \geq\|y\|^{2}-2\langle x, J y\rangle+\|x\|^{2}-\left(1-c_{1}\right)\|x\|^{2} \\
\|x-y\|^{2} & \geq \phi(x, y)-\left(1-c_{1}\right)\|x\|^{2} \\
\|x-y\|^{2} & \geq \phi(x, y)+\left(c_{1}-1\right)\|x\|^{2}
\end{aligned}
$$

Lemma 2.5. Let $E$ be a 2-uniformly smooth real Banach space. Then there exists $c_{2}>0$ such that

$$
\|x-y\|^{2} \geq \phi(x, y)-c_{2}\|x\|^{2} \forall x, y \in E .
$$

Proof. By Theorem 2.2, we have

$$
\|x+y\|^{2} \leq\|x\|^{2}+2\langle y, J x\rangle+c\|y\|^{2} .
$$

Interchanging $x$ and $y$, we obtain

$$
\|x+y\|^{2} \leq\|y\|^{2}+2\langle x, J y\rangle+c\|x\|^{2} .
$$

Replacing $y$ by $(x+y)$ and $x$ by $(-x)$ we get

$$
\|y\|^{2} \leq\|x+y\|^{2}-2\langle x, J(x+y)\rangle+c\|x\|^{2} .
$$

Which implies

$$
\begin{aligned}
\|x+y\|^{2} & \geq\|y\|^{2}+2\langle x, J(x+y)\rangle-c\|x\|^{2} \\
& =\left(\|x\|^{2}+2\langle x, J(x+y)\rangle+\|y\|^{2}\right)+2\langle x, J y\rangle-2\langle x, J y\rangle-c_{2}\|x\|^{2},
\end{aligned}
$$

with $c_{2}=c+1$. Replacing $y$ by $(-y)$ and using the fact that the normalized duality map is monotone, we obtain:

$$
\begin{aligned}
\|x-y\|^{2} & \geq\left(\|x\|^{2}-2\langle x, J y\rangle+\|y\|^{2}\right)+2[\langle x, J y\rangle-2\langle x, J(y-x)\rangle]-c_{2}\|x\|^{2} \\
& =\phi(x, y)+2[\langle x, J y\rangle-\langle x, J(y-x)\rangle]-c_{2}\|x\|^{2} \\
& \geq \phi(x, y)-c_{2}\|x\|^{2}
\end{aligned}
$$

establishing the Lemma.

Lemma 2.6. Let $E$ be a smooth and 2-uniformly convex real Banach space. Then there exists $c>0$ such that, the following inequality holds:

$$
\left\|w_{1}-w_{2}\right\|^{2} \geq \psi\left(w_{1}, w_{2}\right)-c\left\|w_{1}\right\|^{2}, w_{1}=\left(x, x^{*}\right) \in E \times E^{*}, w_{2}=\left(y, y^{*}\right) \in E \times E^{*} .
$$

Proof. Since $E$ is 2-uniformly convex, then $E^{*}$ is 2-uniformly smooth. Using Lemma 2.4 and Lemma 2.5, we have:

$$
\begin{aligned}
\|x-y\|^{2}+\left\|x^{*}-y^{*}\right\|^{2} & \geq \phi(x, y)+\left(c_{1}-1\right)\|x\|^{2}+\phi_{*}\left(x^{*}, y^{*}\right)-c_{2}\left\|x^{*}\right\|^{2} \\
\left\|w_{1}-w_{2}\right\|^{2} & \geq \psi\left(w_{1}, w_{2}\right)-c\left\|w_{1}\right\|^{2} .
\end{aligned}
$$

where $c=\max \left\{1-c_{1}, c_{2}\right\}$.

Lemma 2.7 (Kamimura and Takahashi[30]). Let $E$ be a real smooth Banach space and uniformly convex space, and let $\left\{x_{n}\right\}$ and $\left\{y_{n}\right\}$ be two sequences of E. If either $\left\{x_{n}\right\}$ or $\left\{y_{n}\right\}$ is bounded and $\phi\left(x_{n}, y_{n}\right) \rightarrow 0$ as $n \rightarrow \infty$, then $\left\|x_{n}-y_{n}\right\| \rightarrow 0$ as $n \rightarrow \infty$.

Lemma 2.8 (Tan and $\mathrm{Xu}[36])$. Let $\left\{a_{n}\right\}$ be a sequence of non-negative real numbers satisfying the following relation:

$$
a_{n+1} \leq a_{n}+\sigma_{n} \quad \forall n \geq 0
$$

Assume that $\sum_{n=0}^{\infty} \sigma_{n}<\infty$. Then $\lim _{n \rightarrow \infty} a_{n}$ exists. 
We prove the following results.

Theorem 3.3. For $q>1$, let $E$ be a 2-uniformly convex and q-uniformly smooth real Banach space with dual $E^{*}$ and let $F: E \rightarrow E^{*}, K: E^{*} \rightarrow E$ be bounded and strongly monotone mappings with $D(K)=R(F)=E^{*}$. For given $u_{1} \in E$ and $v_{1} \in E^{*}$, let $\left(\left\{u_{n}\right\}\right.$ and $\left\{v_{n}\right\}$ be generated iteratively by:

$$
\begin{array}{ll}
u_{n+1}=J^{-1}\left(J u_{n}-\alpha_{n}\left(F u_{n}-v_{n}\right)\right), & n \geq 1, \\
v_{n+1}=J\left(J^{-1} v_{n}-\alpha_{n}\left(K v_{n}+u_{n}\right)\right), & n \geq 1,
\end{array}
$$

where $\left\{\alpha_{n}\right\} \subset(0,1)$ satisfies the following conditions: $(i) \sum_{n=1}^{\infty} \alpha_{n}=\infty ;(i i) \sum_{n=0}^{\infty} \alpha_{n}^{2}<\infty$. Suppose that the equation $u+K F u=0$ has a solution. Then, there exists $\gamma_{0}>0$ such that if $\alpha_{n}<\gamma_{0} \forall n \geq 1$, the sequence $\left\{u_{n}\right\}$ converges strongly to $u^{*}$, a solution of $u+K F u=0$.

Proof. Let $X=E \times E^{*}$ with the norm $\|z\|=\left(\|u\|^{2}+\|v\|^{2}\right)^{\frac{1}{2}}$, where $z=(u, v) \in E \times E^{*}$. Define the sequence $\left\{w_{n}\right\}$ in $X$ by: $w_{n}=\left(u_{n}, v_{n}\right)$. Let $u^{*} \in E$ be a solution of $u+K F u=0$. Set $v^{*}:=F u^{*}$ and $w^{*}:=\left(u^{*}, v^{*}\right)$. We observe that $u^{*}=-K v^{*}$.

In the following, we denote by $k_{1}$ the strongly monotonocity constant of $F, k_{2}$ is the strongly monotonocity constant of $K$ and $L_{1}$ the Lipschitz constant of $J^{-1}$. The proof will be in two steps.

Step 1: We first prove that $\left\{w_{n}\right\}$ is bounded. There exists $r>0$ such that:

$$
r \geq \max \left\{4 c\left\|w^{*}\right\|^{2}, \psi\left(w^{*}, w_{1}\right)\right\} .
$$

We show that $\psi\left(w^{*}, w_{n}\right) \leq r$ for all $n \geq 1$. The proof is by induction. We have $\psi\left(w^{*}, w_{1}\right) \leq$ $r$. Assume that $\psi\left(w^{*}, w_{n}\right) \leq r$ for some $n \geq 1$. We show that $\psi\left(w^{*}, w_{n+1}\right) \leq r$. Using the boundedness of $K$, Lemmas 2.1 and 2.2, there exists a positive constant $m_{2}$ such that forall $\alpha \in(0,1),(u, v) \in E \times E^{*}: \psi\left(w^{*},(u, v)\right) \leq r$, the following inequality holds:

$$
\left\|J\left(J^{-1} v-\alpha(K v+u)\right)-J\left(J^{-1} v\right)\right\| \leq \alpha m_{2}\|K v+u\| .
$$

Since $F$ and $K$ are bounded, define

$$
\begin{gathered}
L=\max \left\{L_{1}, m_{2}\right\}, \quad k=\min \left\{k_{1}, k_{2}\right\}, \\
M_{0}=2 L \sup \left\{\|F u-v\|^{2}+\|K v+u\|^{2},(u, v) \in E \times E^{*}, \psi\left(w^{*},(u, v)\right) \leq r\right\}+1, \\
\gamma_{0}=\min \left\{1, \frac{k r}{2 M_{0}}\right\} .
\end{gathered}
$$

Now assume that $\alpha_{n} \leq \gamma_{0}$ for all $n \geq 1$. Using the definition of $u_{n+1}$, we compute as follows:

$$
\begin{aligned}
\phi\left(u^{*}, u_{n+1}\right) & =\phi\left(u^{*}, J^{-1}\left(J u_{n}-\alpha_{n}\left(F u_{n}-v_{n}\right)\right)\right. \\
& =V\left(u^{*}, J u_{n}-\alpha_{n}\left(F u_{n}-v_{n}\right)\right) .
\end{aligned}
$$


Using Lemma 2.3 with $y^{*}=\alpha_{n}\left(F u_{n}-v_{n}\right)$, we have:

$$
\begin{aligned}
\phi\left(u^{*}, u_{n+1}\right)= & V\left(u^{*}, J u_{n}-\alpha_{n}\left(F u_{n}-v_{n}\right)\right) \\
\leq & V\left(u^{*}, J u_{n}\right)-2 \alpha_{n}\left\langle J^{-1}\left(J u_{n}-\alpha_{n}\left(F u_{n}-v_{n}\right)\right)-u^{*}, F u_{n}-v_{n}\right\rangle \\
= & \phi\left(u^{*}, u_{n}\right)-2 \alpha_{n}\left\langle u_{n}-u^{*}, F u_{n}-v_{n}\right\rangle \\
& -2 \alpha_{n}\left\langle J^{-1}\left(J u_{n}-\alpha_{n}\left(F u_{n}-v_{n}\right)\right)-u_{n}, F u_{n}-v_{n}\right\rangle \\
= & \phi\left(u^{*}, u_{n}\right)-2 \alpha_{n}\left\langle u_{n}-u^{*}, F u_{n}-F u^{*}\right\rangle \\
& -2 \alpha_{n}\left\langle J^{-1}\left(J u_{n}-\alpha_{n}\left(F u_{n}-v_{n}\right)\right)-J^{-1}\left(J u_{n}\right), F u_{n}-v_{n}\right\rangle-2 \alpha_{n}\left\langle u_{n}-u^{*}, v^{*}-v_{n}\right\rangle .
\end{aligned}
$$

Using the strong monotonocity of $F$, Schwartz inequality and Lemma 2.1, we obtain

$$
\begin{aligned}
\phi\left(u^{*}, u_{n+1}\right) \leq & \phi\left(u^{*}, u_{n}\right)-2 \alpha_{n} k_{1}\left\|u_{n}-u^{*}\right\|^{2}+ \\
& 2 \alpha_{n}\left\|J^{-1}\left(J u_{n}-\alpha_{n}\left(F u_{n}-v_{n}\right)\right)-J^{-1}\left(J u_{n}\right)\right\|\left\|F u_{n}-v_{n}\right\|+2 \alpha_{n}\left\langle u_{n}-u^{*}, v_{n}-v^{*}\right\rangle \\
\leq & \phi\left(u^{*}, u_{n}\right)-2 \alpha_{n} k_{1}\left\|u_{n}-u^{*}\right\|^{2}+2 \alpha_{n}^{2} L_{1}\left\|F u_{n}-v_{n}\right\|^{2}+2 \alpha_{n}\left\langle u_{n}-u^{*}, v_{n}-v^{*}\right\rangle .
\end{aligned}
$$

We have

(3.29)

$\phi\left(u^{*}, u_{n+1}\right) \leq \phi\left(u^{*}, u_{n}\right)-2 \alpha_{n} k_{1}\left\|u_{n}-u^{*}\right\|^{2}+2 \alpha_{n}^{2} L_{1}\left\|F u_{n}-v_{n}\right\|^{2}+2 \alpha_{n}\left\langle u_{n}-u^{*}, v_{n}-v^{*}\right\rangle$.

Similarly, using the definition of $v_{n+1}$, we compute as follows:

$$
\begin{aligned}
\phi_{*}\left(v^{*}, v_{n+1}\right) & =\phi_{*}\left(v^{*}, J\left(J^{-1} v_{n}-\alpha_{n}\left(K v_{n}+u_{n}\right)\right)\right. \\
& =V_{*}\left(v^{*}, J^{-1} v_{n}-\alpha_{n}\left(K v_{n}+u_{n}\right)\right) .
\end{aligned}
$$

Using Lemma 2.3, with $y^{*}=\alpha_{n}\left(K v_{n}+u_{n}\right)$, we have:

$$
\begin{aligned}
\phi_{*}\left(v^{*}, v_{n+1}\right)= & V_{*}\left(v^{*}, J^{-1} v_{n}-\alpha_{n}\left(K v_{n}+v_{n}\right)\right) \\
\leq & V_{*}\left(v^{*}, J^{-1} v_{n}\right)-2 \alpha_{n}\left\langle J\left(J^{-1} v_{n}-\alpha_{n}\left(K v_{n}+u_{n}\right)\right)-v^{*}, K v_{n}+u_{n}\right\rangle \\
= & \phi_{*}\left(v^{*}, v_{n}\right)-2 \alpha_{n}\left\langle v_{n}-v^{*}, K v_{n}+u_{n}\right\rangle \\
& -2 \alpha_{n}\left\langle J\left(J^{-1} v_{n}-\alpha_{n}\left(K v_{n}+u_{n}\right)\right)-v_{n}, K v_{n}+u_{n}\right\rangle \\
= & \phi_{*}\left(v^{*}, v_{n}\right)-2 \alpha_{n}\left\langle v_{n}-v^{*}, K v_{n}-K v^{*}\right\rangle \\
& \left.-2 \alpha_{n}\left\langle J\left(J^{-1} v_{n}-\alpha_{n}\left(K v_{n}+u_{n}\right)\right)-J\left(J^{-1} v_{n}\right)\right), K v_{n}+u_{n}\right\rangle-2 \alpha_{n}\left\langle u_{n}-u^{*}, v_{n}-v^{*}\right\rangle .
\end{aligned}
$$

Using the strong monotonocity of $K$, Schwartz inequality and inequality (3.25), we obtain

$$
\begin{aligned}
\phi_{*}\left(v^{*}, v_{n+1}\right) \leq & \phi_{*}\left(v^{*}, v_{n}\right)-2 \alpha_{n} k_{2}\left\|v_{n}-v^{*}\right\|^{2}+ \\
& 2 \alpha_{n}\left\|J\left(J^{-1} v_{n}-\alpha_{n}\left(K v_{n}+u_{n}\right)\right)-J\left(J^{-1} v_{n}\right)\right\|\left\|K v_{n}+u_{n}\right\|-2 \alpha_{n}\left\langle u_{n}-u^{*}, v_{n}-v^{*}\right\rangle \\
\leq & \phi_{*}\left(v^{*}, v_{n}\right)-2 \alpha_{n} k_{2}\left\|v_{n}-v^{*}\right\|^{2}+2 \alpha_{n}^{2} m_{2}\left\|K v_{n}+u_{n}\right\|^{2} \\
& -2 \alpha_{n}\left\langle u_{n}-u^{*}, v_{n}-v^{*}\right\rangle .
\end{aligned}
$$

We have

$\phi_{*}\left(v^{*}, v_{n+1}\right) \leq \phi_{*}\left(v^{*}, v_{n}\right)-2 \alpha_{n} k_{2}\left\|v_{n}-v^{*}\right\|^{2}+2 \alpha_{n}^{2} m_{2}\left\|K v_{n}+u_{n}\right\|^{2}-2 \alpha_{n}\left\langle u_{n}-u^{*}, v_{n}-v^{*}\right\rangle$.

Adding up (3.29) and (3.30), using Lemma 2.6 and the definition of $M_{0}$, (3.27), we obtain:

$$
\psi\left(w^{*}, w_{n+1}\right) \leq \psi\left(w^{*}, w_{n}\right)-2 \alpha_{n} k \psi\left(w^{*}, w_{n}\right)+\alpha_{n}^{2} M_{0}+2 k \alpha_{n} c\left\|w^{*}\right\|^{2} .
$$


Finally, using the fact that $\alpha_{n} \leq \gamma_{0}$, the definition of $\gamma_{0}$, (3.28), inequality (3.24) and the induction assumption, it follows that

$$
\begin{aligned}
\psi\left(w^{*}, w_{n+1}\right) & \leq\left(1-2 k \alpha_{n}\right) r+\alpha_{n} k \frac{r}{2}+\alpha_{n} k \frac{r}{2} \\
& \leq\left(1-k \alpha_{n}\left(2-\frac{1}{2}-\frac{1}{2}\right)\right) r \\
& \leq\left(1-k \alpha_{n}\right) r .
\end{aligned}
$$

Therefore, $\psi\left(w^{*}, w_{n+1}\right) \leq r$. Thus, by induction, $\psi\left(w^{*}, w_{n}\right) \leq r$ for all $n \geq 1$. So, from inequality (2.16), we deduce that the sequence $\left\{w_{n}\right\}$ is bounded.

Step 2: We now prove that $\left\{u_{n}\right\}$ converges strongly to $u^{*}$, a solution of $u+K F u=$ 0 . Following the same arguments as in Step 1, using the fact that the sequence $\left\{w_{n}\right\}$ is bounded and the maps $F$ and $K$ are bounded, there exists a positive constant $M$ such that

$$
\psi\left(w^{*}, w_{n+1}\right) \leq \psi\left(w^{*}, w_{n}\right)-2 \alpha_{n} k\left(\left\|v_{n}-v^{*}\right\|^{2}+\left\|u_{n}-u^{*}\right\|^{2}\right)+\alpha_{n}^{2} M .
$$

Therefore,

$$
\psi\left(w^{*}, w_{n+1}\right) \leq \psi\left(w^{*}, w_{n}\right)+\alpha_{n}^{2} M .
$$

Using the assumption $\sum_{n=0}^{\infty} \alpha_{n}^{2}<\infty$ and Lemma 2.8, it follows that $\lim _{n \rightarrow \infty} \psi\left(w^{*}, w_{n}\right)$ exists. From (3.31), we have

$$
\sum_{n=1}^{\infty} \alpha_{n}\left\|w_{n}-w^{*}\right\|^{2}<\infty
$$

Using the fact that $\sum_{n=0}^{\infty} \alpha_{n}=\infty$, we deduce that $\lim \inf \left\|w^{*}-w_{n}\right\|^{2}=0$. Therefore, there exists a subsequence $\left\{w_{n_{k}}\right\}$ of $\left\{w_{n}\right\}$ such that $w_{n_{k}} \rightarrow w^{*}$ as $k \rightarrow \infty$. So, $u_{n_{k}} \rightarrow u^{*}$ and $v_{n_{k}} \rightarrow v^{*}$ as $k \rightarrow \infty$. Using the definitions of $\phi\left(u^{*}, u_{n_{k}}\right)$ and $\phi_{*}\left(v^{*}, v_{n_{k}}\right)$, and the continuity of $J$ and $J^{-1}$, it follows that $\phi\left(u^{*}, u_{n_{k}}\right) \rightarrow 0$ and $\phi_{*}\left(v^{*}, v_{n_{k}}\right) \rightarrow 0$ as $k \rightarrow \infty$. Therefore, from the fact that $\psi\left(w^{*}, w_{n_{k}}\right)=\phi\left(u^{*}, u_{n_{k}}\right)+\phi_{*}\left(v^{*}, v_{n_{k}}\right)$, we have $\psi\left(w^{*}, w_{n_{k}}\right) \rightarrow$ 0 . Since $\lim _{n \rightarrow \infty} \psi\left(w^{*}, w_{n}\right)$ exists, then, $\lim _{n \rightarrow \infty} \psi\left(w^{*}, w_{n}\right)=0$. Thus, $\lim _{n \rightarrow \infty} \phi\left(u^{*}, u_{n}\right)=0$ and $\lim _{n \rightarrow \infty} \phi_{*}\left(v^{*}, v_{n}\right)=0$. From Lemma 2.7, we have $u_{n} \rightarrow u^{*}$ and $v_{n} \rightarrow v^{*}$ as $k \rightarrow \infty$, with $u^{*}$ a solution of the Hammerstein equation. This completes the proof.

Corollary 3.1. For $q>1$, let $E$ be a 2-uniformly convex and $q$-uniformly smooth real Banach space with dual $E^{*}$ and let $F: E \rightarrow E^{*}, K: E^{*} \rightarrow E$ be Lipschitz and strongly monotone mappings with $D(K)=R(F)=E^{*}$. For given $u_{1} \in E$ and $v_{1} \in E^{*}$, let $\left(\left\{u_{n}\right\}\right.$ and $\left\{v_{n}\right\}$ be generated iteratively by:

$$
\begin{array}{ll}
u_{n+1}=J^{-1}\left(J u_{n}-\alpha_{n}\left(F u_{n}-v_{n}\right)\right), & n \geq 1, \\
v_{n+1}=J\left(J^{-1} v_{n}-\alpha_{n}\left(K v_{n}+u_{n}\right)\right), & n \geq 1,
\end{array}
$$

where $\left\{\alpha_{n}\right\} \subset(0,1)$ satisfies the following conditions: ( $\left.i\right) \sum_{n=1}^{\infty} \alpha_{n}=\infty ;(i i) \sum_{n=0}^{\infty} \alpha_{n}^{2}<\infty$. Suppose that the equation $u+K F u=0$ has a solution. Then, there exists $\gamma_{0}>0$ such that if $\alpha_{n}<\gamma_{0} \forall n \geq 1$, the sequence $\left\{u_{n}\right\}$ converges strongly to $u^{*}$, a solution of $u+K F u=0$.

Proof. Since Lipschitz maps are bounded, then the proof follows from Theorem 3.3. 
Corollary 3.2. Let $E=L_{p}, 1<p \leq 2$ and $F: E \rightarrow E^{*}, K: E^{*} \rightarrow E$ be bounded and strongly monotone maps with $D(K)=R(F)=E^{*}$. For given $u_{1} \in E$ and $v_{1} \in E^{*}$, let $\left(\left\{u_{n}\right\}\right.$ and $\left\{v_{n}\right\}$ be generated iteratively by:

$$
\begin{aligned}
& u_{n+1}=J^{-1}\left(J u_{n}-\alpha_{n}\left(F u_{n}-v_{n}\right)\right), \quad n \geq 1, \\
& v_{n+1}=J\left(J^{-1} v_{n}-\alpha_{n}\left(k v_{n}+u_{n}\right)\right), \quad n \geq 1,
\end{aligned}
$$

where $\left\{\alpha_{n}\right\} \subset(0,1)$ satisfies the following conditions: $(i) \sum_{n=1}^{\infty} \alpha_{n}=\infty$; (ii) $\sum_{n=0}^{\infty} \alpha_{n}^{2}<\infty$.

Suppose that the equation $u+K F u=0$ has a solution.Then, there exists $\gamma_{0}>0$ such that if $\alpha_{n}<\gamma_{0} \forall n \geq 1$, the sequence $\left\{u_{n}\right\}$ converges to $u^{*}$, a solution of $u+K F u=0$.

Proof. Since $E=L_{p}$ spaces, $1<p \leq 2$ are 2-uniformly convex and $p$-uniformly smooth Banach spaces, then the proof follows from Theorem 3.3.

Remark 3.2. Using the same method of proof as in Theorem 3.3 by interverting the properties of $J$ and $J^{-1}$, using the duality between $E$ and $E^{*}$, we have the following result.

Theorem 3.4. For $p>1$, let $E$ be a 2-uniformly smooth and $p$-uniformly convex real Banach space with dual $E^{*}$ and let $F: E \rightarrow E^{*}, K: E^{*} \rightarrow E$ be bounded and strongly monotone mappings with $D(K)=R(F)=E^{*}$. For given $u_{1} \in E$ and $v_{1} \in E^{*}$, let $\left(\left\{u_{n}\right\}\right.$ and $\left\{v_{n}\right\}$ be generated iteratively by:

$$
\begin{array}{ll}
u_{n+1}=J^{-1}\left(J u_{n}-\alpha_{n}\left(F u_{n}-v_{n}\right)\right), & n \geq 1, \\
v_{n+1}=J\left(J^{-1} v_{n}-\alpha_{n}\left(K v_{n}+u_{n}\right)\right), & n \geq 1,
\end{array}
$$

where $\left\{\alpha_{n}\right\} \subset(0,1)$ satisfies the following conditions: ( $\left.i\right) \sum_{n=1}^{\infty} \alpha_{n}=\infty ;(i i) \sum_{n=0}^{\infty} \alpha_{n}^{2}<\infty$. Suppose that the equation $u+K F u=0$ has a solution. Then, there exists $\gamma_{0}>0$ such that if $\alpha_{n}<\gamma_{0} \forall n \geq 1$, the sequence $\left\{u_{n}\right\}$ converges strongly to $u^{*}$, a solution of $u+K F u=0$.

Corollary 3.3. Let $E=L_{p}, 2 \leq p<\infty$ and $F: E \rightarrow E^{*}, K: E^{*} \rightarrow E$ be bounded and strongly monotone maps with $D(K)=R(F)=E^{*}$. For given $u_{1} \in E$ and $v_{1} \in E^{*}$, let $\left(\left\{u_{n}\right\}\right.$ and $\left\{v_{n}\right\}$ be generated iteratively by:

$$
\begin{aligned}
& u_{n+1}=J^{-1}\left(J u_{n}-\alpha_{n}\left(F u_{n}-v_{n}\right)\right), \quad n \geq 1, \\
& v_{n+1}=J\left(J^{-1} v_{n}-\alpha_{n}\left(k v_{n}+u_{n}\right)\right), \quad n \geq 1,
\end{aligned}
$$

where $\left\{\alpha_{n}\right\} \subset(0,1)$ satisfies the following conditions: $(i) \sum_{n=1}^{\infty} \alpha_{n}=\infty$; (ii) $\sum_{n=0}^{\infty} \alpha_{n}^{2}<\infty$.

Suppose that the equation $u+K F u=0$ has a solution.Then, there exists $\gamma_{0}>0$ such that if $\alpha_{n}<\gamma_{0} \forall n \geq 1$, the sequence $\left\{u_{n}\right\}$ converges to $u^{*}$, a solution of $u+K F u=0$.

Proof. Since $E=L_{p}$ spaces, $2 \leq p<\infty$ are 2-uniformly smooth and $p$-uniformly smooth Banach spaces, then the proof follows from Theorem 3.4.

Remark 3.3. Real sequences that satisfy conditions $(i)$ and $(i i)$ are given by: $\alpha_{n}=\frac{1}{n}$.

Remark 3.4. The iteration method given by (3.23) is new and is inspired by the one used by Chidume and Djitte [17] in the setting where $F, K: E \rightarrow E$ are accretive type operators.

Acknowledgement. The authors thank the referees for their comments and remarks that helped to improve the presentation of this paper. 


\section{REFERENCES}

[1] Agarwal, R. P., O'Regan, D. and Sahu, D. R., Fixed Point Theory and its applications, Springer, New York, NY, USA, 2009

[2] Alber, Ya., Metric and generalized projection operator in Banach space:properties and applications. In Theory and Applications of Nonlinear Operators of Accretive and Monotone Type (A. G Kartsatos, Ed.), Marcel Dekker, New York (1996), 15-50

[3] Alber, Ya. and Guerre-Delabiere, S., On the projection methods for fixed point problems, Analysis (Munich), 21 (2001), No. 1, 17-39

[4] Alber, Y., Generalized Projection Operators in Banach space: Properties and Applications, Funct. Diferent. Equations, 1 (1994), No. 1, 1-21

[5] Berinde, V., Iterative approximation of fixed points, Lecture Notes in Mathematics 1912, Springer Berlin, 2007

[6] Brézis, H. and Browder, F. E., Existence theorems for nonlinear integral equations of Hammerstein type, Bull. Amer. Math. Soc., 81 (1975), 73-78

[7] Brézis, H. and Browder, F. E, Nonlinear integral equations and systems of Hammerstein type, Advances in math., 18 (1975), 115-147

[8] Brézis., H. and Browder., F. E., Some new results about Hammerstein equations, Bull. Amer. Math. Soc., 80 (1974), 567-572

[9] Browder, F. E., Nonlinear mappings of nonexpansive and accretive type in Banach spaces, Bull. Amer. Math. Soc., 73 (1967), 875-882

[10] Browder, F. E, De Figueiredo, D. G and Gupta, P., Maximal monotone operators and a nonlinear integral equations of Hammerstein type, Bull. Amer. Math. Soc., 76 (1970), 700-705

[11] Browder, F. E. and Gupta, P., Monotone operators and nonlinear integral equations of Hammerstein type, Bull. Amer. Math. Soc., 75 (1969), 1347-1353

[12] Chepanovich, R. Sh., Nonlinear Hammerstein equations and fixed points, Publ. Inst. Math. (Beograd) N. S., 35 (1984), 119-123

[13] Chidume, C. E., Geometric Properties of Banach spaces and Nonlinear Iterations, Springer Verlag Series: Lecture Notes in Mathematics, 1965 (2009), ISBN 978-1-84882-189-7

[14] Chidume, C. E. and Djitte, N., An iterative method for solving nonlinear integral equations of Hammerstein type, Appl. Math. Comput., 219 (2013), 5613-5621

[15] Chidume, C. E. and Djitte, N., Approximation of Solutions of Nonlinear Integral Equations of Hammerstein Type, ISRN Mathematical Analysis, 2012, Article ID 169751, 12 pp. doi:10.5402/2012/169751

[16] Chidume, C. E and Djitte, N., Convergence Theorems for Solutions of Hammerstein Equations with Accretive-Type Nonlinear Operators, Panamer. Math. J., 22 (2012), No. 2, 19-29

[17] Chidume., C. E. and Djitte, N., Approximation of solutions of Hammerstein equations with bounded strongly accretive nonlinear operators, Nonlinear Anal., 70 (2009), 4071-4078

[18] Chidume, C. E. and Djitte, N., Iterative approximation of solutions of Nonlinear equations of Hammerstein type, Nonlinear Anal., 70 (2009), 4086-4092

[19] Chidume, C. E. and Ofoedu, E. U., Solution of nonlinear integral equations of Hammerstein type, Nonlinear Anal., 74 (2011), 4293-4299

[20] Chidume, C. E. and Osilike, M. O., Iterative solution of nonlinear integral equations of Hammerstein type, J. Nigerian Math. Soc., 11 (1992), 9-18, MR96c:65207

[21] Chidume, C. E. and Shehu, Y., Approximation of solutions of generalized equations of Hammerstein type, Computer Math. Appl., 63 (2012), 966-974

[22] Chidume, C. E. and Zegeye, H., Approximation of solutions of nonlinear equations of Hammerstein type in Hilbert space, Proc. Amer. Math. Soc., 133 (2005), No. 3, 851-858

[23] Chidume, C. E. and Zegeye, H., Approximation of solutions of nonlinear equations of monotone and Hammerstein type, Appl. Appl. Anal., 82 (2003), No. 8, 747-758

[24] Chidume, C. E. and Zegeye, H., Iterative approximation of solutions of nonlinear equations of Hammerstein type, Abstr. Appl. Anal., 6 (2003), 353-367

[25] De Figueiredo, D. G. and Gupta, C. P., On the variational method for the existence of solutions to nonlinear equations of Hammerstein type, Proc. Amer. Math. Soc., 40 (1973), 470-476

[26] Djitte, N. and Sene, M., An Iterative Algorithm for Approximating Solutions of Hammerstein Integral Equations, Numer. Funct. Anal. Opti., 34 (2013), No. 12, 1299-1316

[27] Djitte, N. and Sene, M., Approximation of Solutions of Nonlinear Integral Equations of Hammerstein Type with Lipschitz and Bounded Nonlinear Operators, ISRN Applied Mathematics, 2012, Article ID 963802, 15 pp. doi:10.5402/2012/963802

[28] Dolezale, V., Monotone operators and its applications in automation and network theory, Studies in Automation and Control 3, (Elsevier Science Publ. New York, 1979)

[29] Hammerstein, A., Nichtlineare integralgleichungen nebst anwendungen, Acta Math. Soc., 54 (1930), 117-176 
[30] Kamimura, S. and Takahashi, W., Strong convergence of proximal-type algorithm in Banach space, SIAMJ. Optim., 13 (2002), No. 3, 938-945

[31] Kato, T., Nonlinear semi groups and evolution equations, J. Math. Soc. Japan, 19 (1967), 508-520

[32] Mann, W. R., Mean value methods in iteration, Proc. Amer. Math. Soc., 4 (1953), 506-510

[33] Matouskova, E. and Reich, S., Projection and proximal point methods: convergence results and counter examples, Nonlinear Anal., 56 (2004), 715-738

[34] Pascali, D. and Sburlan, S., Nonlinear mappings of monotone type, Editura Academiei, Bucureşti, Romania (1978)

[35] Reich, S., Constructive techniques for accretive and monotone operators, Applied non-linear analysis, Academic Press, New York (1979), 335-345

[36] Tan, H. K. and Xu, H. K., Approximating fixed points of nonexpansive mappings by the Ishikawa iteration process, J. Math. Anal. Appl, 178 (1993), No. 2, 301-308

[37] Xu, H. K., Inequalities in Banach spaces with applications, Nonlinear Anal., 16 (1991), No. 12, 1127-1138

[38] Zeidler, E., Nonlinear functional analysis and its applications, Part II: Monotone operators, Springer-Verlag, Berlin/New York, 1985

DEPARTMENT MATHEMATICS

GASTON BERGER UNIVERSITY

BP 234, SAINT LOUIS, SENEGAL

Email address: sowthierno89@gmail.com

Email address: diopmotors@hotmail.com

Email address: ngalla.djitte@ugb.edu.sn 